\title{
Pacientes com Câncer Avançado: 0 Acesso aos Opioides e demais Medicamentos para Controle da Dor
}

doi: https://doi.org/10.32635/2176-9745.RBC.2018v64n2.78

Patients with Advanced Cancer: the Access to Opioids and other Pain Control Medication
Pacientes con Cáncer Avanzado: el Acceso a los Opioides y otros Medicamentos para el Control del Dolor

Francine Rosa Portela'; Celina Maria Modena²

Resumo

Introduçáo: A dor é o sintoma mais frequente e grave vivenciado pelos pacientes com câncer, acarretando sofrimento e incapacidades. Objetivo: Descrever a percepção dos familiares de pacientes com câncer avançado quanto às dificuldades no acesso aos opioides e a outros medicamentos para dor. Método: A pesquisa se insere na perspectiva da abordagem qualitativa. Foram entrevistados 14 cuidadores informais, familiares das pessoas com diagnóstico de câncer em estágio avançado com potencial de finitude, sendo utilizadas entrevistas semiestruturadas. Resultados: Todos os participantes relataram comprar os medicamentos para dor; entre eles, os opioides e outros medicamentos para demais sintomas dos seus familiares com câncer avançado, impactando na renda familiar. O alívio do sofrimento severo relacionado à saúde deve ser prioritário, um imperativo ético que transversalize a assistência aos pacientes com câncer e demais adoecimentos que ameaçam ou limitam a vida. Conclusão: É necessário o avanço no Programa de Políticas Públicas para os pacientes com câncer, no qual seja prioritário o eficaz controle da dor, pautado na facilitação do acesso, na dispensação dos opioides e demais medicamentos para controle da dor. Contudo, a garantia do acesso deve ser alicerçada para a formação médica e avaliação eficaz, e adequada prescrição no controle da dor, de acordo com os recursos disponíveis. Somente desse modo, se intervirá no sofrimento, garantindo a equidade e a promoção da dignidade humana para os que vivenciam a dor.

Palavras-chave: Cuidados Paliativos; Neoplasias; Dor; Analgésicos Opioides; Direitos Humanos.

\section{Abstract}

Introduction: Pain is the worst and most frequent symptom of cancer patients causing suffering and inabilities. Objective: To describe how family relatives of patients with advanced cancer perceive the difficulties of accessing opioids and other pain medication. Method: This research adopts a Qualitative "Approach". Fourteen informal caretakers, family members of people diagnosed with advanced cancer, were interviewed through semi structured interviews. Results: All participants reported that they bought pain medication, including opioids and other drugs for cancer symptoms, for their relatives with advanced cancer, which had some impact on the family income. The relief of extreme suffering related to health must be a priority. It must be an ethical imperative that cuts across the assistance to patients with advanced cancer and other life threatening diseases. Conclusion: Public Health Programs for patients with advanced cancer must be enhanced in order to prioritize the easy access to opioids and other pain control drugs. Nonetheless, the guarantee of access must be held at medical discretion, with the accurate evaluation and prescription of pain control methods, considering the available resources. Only then it will be possible to ease suffering, ensuring equity and promoting human dignity for the ones who experience pain.

Key words: Palliative Care; Neoplasms; Pain; Analgesics, Opioids; Human Rights.

\section{Resumen}

Introducción: El dolor es el síntoma más frecuente y grave vivido por los pacientes con cáncer, acarreando sufrimiento e incapacidades. Objetivo: Describir la percepción de los familiares de pacientes con cáncer avanzado en cuanto a las dificultades en el acceso a los opioides y otros medicamentos para el dolor. Método: La investigación se inserta en la perspectiva del Enfoque cualitativo. Se entrevistaron a 14 cuidadores informales, familiares de las personas con diagnóstico de cáncer en estadio avanzado con potencial definición, siendo utilizadas entrevistas semiestructuradas. Resultados: Todos los participantes relataron comprar los medicamentos para el dolor, entre ellos los opioides y otros medicamentos para los demás síntomas de sus familiares con cáncer avanzado, impactando la renta familiar. El alivio del sufrimiento severo relacionado a la salud debe ser prioritario, un imperativo ético que transversaliza la asistencia a los pacientes con cáncer y demás adoecimentos que amenazan o limitan la vida. Conclusión: Es necesario el avance en el Programa / Política Pública para los pacientes con cáncer donde sea prioritario el eficaz control del dolor, pautado en la facilitación del acceso, la dispensa de los opioides y demás medicamentos para control del dolor. Sin embargo, la garantía del acceso debe ser establecida a la formación médica para una evaluación eficaz y adecuada prescripción en el control del dolor, de acuerdo con los recursos disponibles. Sólo de este modo se intervendrá en el sufrimiento, garantizando la equidad y promoción de la dignidad humana para los que experimentan el dolor.

Palabras clave: Cuidados Paliativos; Neoplasias; Dolor; Analgésicos Opioides; Derecho Humano.

\footnotetext{
${ }^{1}$ Psicóloga Oncológica. Grupo de Pesquisa de Políticas Públicas e Direitos Humanos em Saúde e Saneamento (PPDH). Instituto René Rachou (IRR). Fundação Oswaldo Cruz (Fiocruz)/Minas. Belo Horizonte (MG). Brasil. E-mails: francineportela@hotmail.com/francine.portela@cpqrr.fiocruz.br. Orcid ID: https://orcid. org/0000-0003-0299-112X

2 Psicóloga. Pós-Doutorado em Saúde Coletiva pelo IRR/Fiocruz Minas. PPDH. Belo Horizonte (MG), Brasil. E-mail: celina@minas.fiocruz.br. Orcid ID: https://orcid. org/0000-0001-5035-3427
}

Endereço para correspondência: Celina Maria Modena. Av. Augusto de Lima, 1715 - Barro Preto. Belo Horizonte (MG), Brasil. CEP $33190-002$. 


\section{INTRODUÇÃO}

Recentemente, a Revista The Lancet publicou uma pesquisa, fruto da investigaçáo de três anos, realizada pela Comissão Lancet sobre atenuação de acesso ao abismo em cuidados paliativos e alívio da dor. A publicação é um relatório que evidencia a carga de sofrimento negligenciada no mundo. Estima-se que, mundialmente, 61 milhóes de pessoas, incluindo os que morrem por ano e os que vivem em condiçôes de saúde que ameacem ou limitem a vida, são afetadas por sofrimentos relacionados à saúde; destas, 2,5 milhóes são crianças ${ }^{1}$.

De acordo com a publicação, $45 \%$ dos que morrem por ano vivenciam sofrimento grave. Destes, mais de $80 \%$ não receberam alívio da dor e cuidados paliativos constituindo "Um Mar de Sofrimento"2.

A negligência dos sofrimentos severos relacionados à saúde "é uma falha médica, de Saúde Pública e moral, e uma paródia de justiça”. Cuidados paliativos e alívio da dor devem ser elementos essenciais da cobertura universal de saúde (p.1391) ${ }^{1}$.

A necessidade de alívio da dor e cuidados paliativos tem sido negligenciada mundialmente. Atualmente, há uma grande valorização da ampliação do tempo de vida, da produtividade, com pouco peso para as intervençóes que aliviem a dor, o sofrimento ou o aumento da dignidade no final da vida.

A Associação Latino-Americana de Cuidados Paliativos (ALCP), com base na Organização Mundial da Saúde (OMS), preconiza que o modelo de Saúde Pública para o desenvolvimento dos Cuidados Paliativos deve ser composto por políticas públicas adequadas, alicerçadas na educação, capacitação em cuidados paliativos e no acesso aos opioides e medicamentos essenciais ${ }^{3}$. Esses dois alicerces (educação e acesso aos medicamentos) são a base para a implantação dos cuidados paliativos em todos os níveis de atençấo do sistema de saúde.

A OMS conceitua os cuidados paliativos como:

uma abordagem que melhora a qualidade de vida dos pacientes (adultos e crianças) e suas famílias que enfrentam problemas associados a doenças potencialmente fatais. Previne e alivia o sofrimento através da identificação precoce, avaliação correta e tratamento da dor e outros problemas, sejam eles físicos, psicossociais ou espirituais ${ }^{4}$.

Os cuidados paliativos possuem como princípios promover a qualidade de vida e a dignidade humana, sendo imprescindível para tal a avaliaçâo adequada e controle da dor.

De acordo com o Instituto Nacional de Câncer José Alencar Gomes da Silva (INCA), a dor é o principal sintoma do paciente com câncer em progressão, acarretando sofrimento e incapacidades 5 . É um dos sintomas mais frequentes e graves vivenciados pelos pacientes que necessitam de cuidados paliativos. Os opioides são essenciais para o tratamento da dor, além de tratar outros sintomas angustiantes como a falta de ar. A OMS estima que $80 \%$ dos pacientes com câncer ou Aids sofrerão de dor moderada a grave no fim da vida, sendo indispensável o uso dos opioides ${ }^{4}$. Contudo, para a OMS, o controle dos sintomas não se restringe ao final de vida, sendo uma obrigação ética para o alívio do sofrimento e respeito à dignidade humana o controle dos sintomas desde o diagnóstico, independente do estágio ser inicial ou avançado da doença.

No Consenso Brasileiro sobre Manejo da Dor Relacionada ao Câncer, o controle efetivo da dor deve ser prioritário no tratamento oncológico por contribuir para uma melhor tolerância do paciente ao tratamento do câncer e por mais tempo, somado ao impacto positivo na qualidade de vida e na sobrevida ${ }^{6}$.

Segundo o INCA, preparar a equipe é a principal estratégia para controle da dor e demais sintomas na assistência aos pacientes com câncer avançado.

É condição imprescindível que os profissionais de saúde saibam como controlar a dor de pacientes com câncer avançado, que reajam contra mitos e conceitos principais sobre as drogas disponíveis e que se mantenham atualizados com foco no tratamento da dor adequado aos recursos disponíveis ${ }^{5(6)}$.

Um ponto principal para qualidade na assistência em cuidados paliativos é garantir o acesso aos opioides ${ }^{7}$. $\mathrm{O}$ Brasil, na última década, adotou medidas para facilitar o acesso aos opioides, tais como: simplificação da prescrição e facilitação de uso de receituários para prescrição de opioides; revisão da Relaçáo Nacional de Medicamentos Essenciais (Rename), por meio da Portaria GM/MS 1.587 de 3 de setembro de 2002; inclusão da morfina, metadona e codeína no Programa de Medicamentos Excepcionais, garantindo a disponibilização gratuita para a população necessitada e ampliação do acesso (Portaria GM/MS 1.318 de 23 de julho de 2001); estabelecimento de um Protocolo Clínico e Diretrizes Terapêuticas para o uso de Opioides no Alívio da Dor Crônica ${ }^{8}$.

Apesar do avanço, advindo principalmente de intervençóes normativas, como as Portarias, o controle da dor ainda é precário no Brasil, sendo o maior obstáculo a pouca informação e formação de profissionais de saúde e dos gestores locais sobre os cuidados paliativos ${ }^{8}$.

A Organização para Prevenção de Sofrimento Intenso (OPIS), em um evento oficial, paralelo a $37^{\text {a }}$ sessão do Conselho de Direitos Humanos da Organização das 
Nações Unidas (ONU), abordou a situação devastadora do sofrimento intenso pelo acesso negado à morfina. Com o título "Por fim a agonia: o acesso à morfina como imperativo ético e de direitos humanos"(1), o movimento realizado pela OPIS traz à tona a dificuldade do acesso à morfina e a outros opiáceos. Segundo a Organização, a dificuldade deve-se, em grande parte, às regulaçóes governamentais excessivas, baseadas em preocupaçóes desproporcionais sobre a dependência e o abuso, assim como a histórica negligência em relação ao sofrimento cometida por muitos sistemas de saúde?

Em nível global, a OMS nomeia diversos obstáculos para atender às necessidades insatisfatórias em cuidados paliativos; entre elas, está o acesso da população aos opioides, que é insuficiente e não se encontra em conformidade com as convençóes internacionais sobre acesso a medicamentos essenciais ${ }^{4}$.

\section{MÉTODO}

A referida pesquisa se insere na perspectiva da abordagem qualitativa e possui como objetivo descrever a percepção dos familiares de pacientes com câncer avançado sobre o acesso aos opioides e demais medicamentos para dor.

Optou-se pela Abordagem Qualitativa por causa da potencialidade de explorar o que não pode ser quantificável, utilizando o significado atribuído pelos sujeitos às práticas, às aspiraçôes, aos valores e atitudes ${ }^{10}$.

Foram entrevistados 14 cuidadores informais de pessoas com diagnóstico de câncer em estágio avançado. Compreendem-se cuidadores informais os familiares, as pessoas com vínculos de parentesco de família com o paciente e as pessoas com vínculos afetivos com o doente e que não sejam familiares. Os critérios de inclusão foram: ter idade igual ou superior a 18 anos, ser o cuidador de referência do paciente, estar como cuidador desse paciente há no mínimo um mês, concordar em participar da pesquisa. $\mathrm{O}$ número de participantes obedeceu ao critério de saturação do discurso, no qual se encerra a inclusão de participantes ao observar-se a repetição das narrativas coletadas ${ }^{11}$.

A referida pesquisa foi aprovada pelos Comitês de Ética do Centro de Pesquisas René Rachou/Fundação Oswaldo Cruz, sob o número de parecer 2.360.868 (CAAE: 76425617.9.0000.5091) e do Hospital de Clínicas da Universidade Federal do Triângulo Mineiro (HC/UFTM), número 2.430.351 (CAEE: 76425617.9.3001.5154).

Foram realizadas 14 entrevistas semiestruturadas, com duração média de 50 minutos. Optou-se por essa técnica por permitir a coleta de dados objetivos e subjetivos e facilitar o caráter relacional, maior proximidade entre o entrevistador e o entrevistado, possibilitando discorrer com profundidade sobre o tema proposto ${ }^{12}$, dado o assunto complexo, objeto do referido estudo: experiência, vivências e percepçóes dos cuidadores principais quanto ao acesso a opioides e a medicamentos para controle da dor. As entrevistas foram realizadas no HC/UFTM, no espaço do ambulatório ou enfermaria, no horário de escolha do participante (cuidador familiar).

Para a inclusão dos participantes, o projeto foi apresentado às equipes do HC/UFTM, que atendem aos pacientes com câncer, e solicitado que os profissionais sugerissem potenciais participantes, de acordo com o critério de inclusão. Os possíveis participantes foram abordados pessoalmente pelo pesquisador, sendo apresentados a eles a pesquisa e seus objetivos, o TCLE, e averiguado se teriam interesse em participar do estudo.

Foi garantida ao participante a confidencialidade da entrevista e este foi informado que seria identificado na pesquisa apenas pelo seu vínculo familiar com o paciente, conforme descrito no Termo de Consentimento Livre e Esclarecido (TCLE).

As entrevistas semiestruturadas foram realizadas com base nas seguintes perguntas: Recentemente o seu familiar (o paciente) apresentou dor? Se sim, houve atendimento para essa dor? Em sua opiniáo, o atendimento recebido ajudou a aliviar esse sintoma? O seu familiar toma ou já tomou algum medicamento para dor, como morfina, codeína, outros? Vocês compraram esse(s) medicamento(s) ou o(s) conseguiram pelo SUS?

A coleta dos dados (entrevistas) foi realizada de dezembro/2017 a janeiro/2018. Todas as entrevistas foram gravadas e transcritas. A análise de dados das 14 entrevistas foi realizada na perspectiva da Análise de Conteúdo ${ }^{13}$.

\section{RESULTADOS E DISCUSSÃO}

A dor tem sido considerada como um dos principais sintomas no impacto da qualidade de vida dos pacientes com câncer. No universo da pesquisa, $71 \%$ dos participantes relataram que os pacientes apresentaram dor. Entretanto, deve ser salientado que, caso os participantes fossem os pacientes e não os familiares, esse resultado possivelmente seria diferente, pois, em estudo clássico, $70 \%$ dos familiares hiperestimaram a dor do paciente ${ }^{14}$.

As narrativas a seguir apontam que o acesso aos opioides via Sistema Único de Saúde (SUS) ainda é inacessível para a populaçấo estudada:

Não, a gente nem chegou a tentar [pelo SUS] [...] não é fácil conseguir pelo SUS. [...] E entâo vai juntando aos poucos, e tem vezes que não dá para comprar e aí a gente dá um jeito (P10, filha).

Para dor, ela estava somente com buscopan e tem um tal de um Paco que eu não sei se é a mesma 
coisa. Eu comprei. Porque pelo SUS eles não têm (P1, filha).

Agora ela toma um outro medicamento que a gente consegue comprar [...] Codeína. [...] A gente compra. Porque a gente recebeu a orientação de que a gente não conseguiria. Porque ele é muito em conta mesmo. E ela tem o auxílio [benefício social] e então a gente consegue comprar (P8, filha).

Uai, um pouco [dos medicamentos] é do SUS, um pouco ela compra. [...] porque ela compra na farmácia por mês, quando ela recebe, ela vai lá e paga. [...] Só sei que é caro (P13, filho).

Apesar de as normativas (portarias) garantirem o acesso gratuito aos medicamentos para os pacientes com dor crônica pelo SUS, na vivência dos participantes, esse acesso não foi garantido, cabendo aos familiares e pacientes a compra dos medicamentos, impactando a renda familiar.

Um obstáculo também para o acesso é a farmácia que disponibilize morfina e seus derivados. Conforme a narrativa, a seguir, de um participante, uma dificuldade nos municípios menores é a indisponibilidade para a compra dos medicamentos nas farmácias.

O medicamento ela toma vários e até caros. Hoje mesmo eu vou ter que comprar daqui para levar [do município de grande porte], têm muitos que não consegue não [no município em que reside] (P14, pai).

No Brasil, a comercialização de medicamentos de uso controlado é alvo de intensa fiscalizaçáo e de processos burocratizados por parte das autoridades, causando dificuldades para as farmácias particulares, que, muitas vezes, optam por não oferecer esses medicamentos, dificultando o acesso à população ${ }^{15}$.

Conforme relato do participante, a dificuldade para compra do medicamento morfina torna-se mais complexa para os pacientes que náo residem nas capitais, ou cidades grandes. Ressalta-se que a distância entre o município onde "P14" reside e o município onde conseguiu comprar o medicamento é de aproximadamente $310 \mathrm{~km}$, em torno de cinco horas de viagem.

Os participantes apontaram dificuldades em atender aos trâmites de acesso a medicamentos, o que pode ser evidenciado na narrativa abaixo.

No começo sim, por causa desta documentação que tinha que fazer [...] Ela tomou o tramal [tramadol]. [...] Demorou [para conseguir o medicamento] eu acho que umas três semanas depois que ela foi para casa [...] você vai na prefeitura e aí você vai na assistente social e é um medicamento mais carinho mesmo. Então eles foram lá em casa fizeram uma visita e entrevistaram a gente. Foi todo este procedimento para poder liberar [o tramadol] (P8, filha).

No Brasil, a gestão dos recursos para opioides é descentralizada, dependente da estrutura sanitária dos Estados e municípios ${ }^{8}$. Na maioria das localidades, o gestor local não possui um programa específico com registro dos usuários que utilizam o medicamento, o que facilitaria a distribuição. Assim, a prescriçấo requer receituários especiais e diversos formulários. A desinformação das unidades que dispensam esses medicamentos também contribui para dificultar o processo.

Dos participantes, $14 \%$ referem conseguir medicamentos por meio de doaçôes:

Pelo posto não. Eu consigo nestas farmácias que recebem doação. Igual o Hospital X tem uma farmácia também lá na região que tem um medicamento dele que eu consigo ganhar lá às vezes. Mas os outros medicamentos a gente tem que comprar. A Dipirona é o único remédio que eu consigo ganhar para ele pelo SUS. Eu vou na farmacinha e consigo ganhar alguma coisa. O dimorf eu não precisei de comprar ele, eu consigo ganhar ele na farmacinha, quando eu preciso (P5, esposa).

Não chega a ser uma ONG, mas tem uma farmácia que doou uns remédios para ela, que precisava. Estes remédios para vomito: Isso, aqui perto do hospital. Foi o pessoal daqui mesmo que indicou para a gente. [...] Pertinho daqui, eles indicaram e a gente foi lá e conseguiu o remédio que precisava no momento. [...] Era um remédio para enjoo (P8, filha).

Uma pesquisa publicada pela The Economist salienta a importância da participação de instituições não governamentais na provisão de apoio e assistência em Cuidados Paliativos ${ }^{16}$.

As doaçôes, segundo o relato dos participantes, possibilitam um caminho para conseguir alguns medicamentos e minimizar o impacto nos custos da renda familiar:

Assim, com remédio a gente gasta no mínimo assim, eu conseguindo ganhar o dimorf, conseguindo ganhar a Dipirona, eu gasto mais ou menos uns $\mathrm{R} \$$ 150,00 com os outros dois remédios por mês. E aí quando eu náo ganho e preciso comprar aí são uns $\mathrm{R} \$ 300,00$. Porque o dimorf é caro, e o outro nem é tanto, mas o dimorf é bem caro e a Dipirona também e aí eu gasto mais por causa destes dois (P5, esposa).

Um dos aspectos levados em consideração na avaliaçáo do desenvolvimento dos cuidados paliativos nos países é o investimento do governo. Quando não 
há tal investimento, ocorre a sobrecarga financeira para os pacientes, familiares ${ }^{7}$. Embora existam no Brasil normativas que garantam o acesso aos medicamentos para dor, há uma fragilidade no acesso para a população estudada.

A inacessibilidade aos opioides e demais medicamentos essenciais para controle de sintomas dos pacientes com câncer acarreta em um grande impacto na renda das famílias, já, em boa parte, comprometidas com as necessidades básicas, como a alimentação, conforme esta narrativa:

Eu vou te falar uma coisa, já era pouco e agora está mais esticado ainda, porque só meu é $\mathrm{R} \$ 180,00$ todo mês de remédio [participante é cardiopata, decorrente da doença de chagas]. Para ela [...] já gastei R \$ 420,00 de remédio. [...] Então, para quem ganha um salário, tem que pagar mais $\mathrm{R} \$ 108,00$ de água, mais $\mathrm{R} \$ 86,00$ de energia, sobra pouco para comer. [...] às vezes, falta um páo, às vezes, falta uma verdura, tem dias que, às vezes, falta uma carne também, porque não é todo dia que você dá conta. Entáo, o cabra vai regulando (P7, marido).

Uma participante relata que os remédios indispensáveis para o paciente são comprados, o que, somado aos custos básicos para manter a casa, impacta financeiramente no mês, impossibilitando para investirem em uma boa alimentação:

Nós vivemos com $R \$ 1.200,00$ e também tem a alimentação dele que precisa de muita verdura e isso a gente não tem onde ganhar. Eu preciso comprar e, muitas vezes, fica em falta em casa nesta parte, por não ter como comprar e ele precisa de comer e a gente náo tem. [...] Mas a gente náo consegue fazer uma alimentaçấo muito boa, porque preocupa muito com conta, a gente tem que gastar mais com isso, com conta, porque tem água e luz e a gente não pode ficar sem. [...] E aí preciso do gás para fazer a comida e a gente vive de aluguel. E entấo a maior parte do dinheiro dele fica para a despesa mesmo. E aí o que sobra para o remédio e aí depois eu vou me virando. [...] geralmente faço um bico e aí é onde eu consigo comprar a verdura e a fruta ( $\mathrm{P} 5$, esposa).

Os medicamentos para controle da dor e demais sintomas, e a alimentação foram as principais preocupaçôes narradas pelos participantes da pesquisa, sendo um gasto custeado pelas famílias.

Outro aspecto que os participantes relataram foi o retorno à unidade de saúde (pronto-socorro, emergência, posto de saúde), por diversas vezes, em razão da dor constante do seu familiar.
Olha... tinha noite que eu trazia ela até três vezes [na emergência do hospital]. Porque ela tomava o remédio aliviava, e mandavam ela embora [para casa]. Aí eu levava ela para casa. Chegava lá ela dormia um sono, acordava com dor. Aí náo tinha remédio para dar, não tinha um remédio para cortar a dor. Eu trazia ela de novo na mesma noite. Teve uma noite que eu trouxe ela três vezes [para a emergência] (P7, marido).

Ela foi no posto de saúde e no posto de saúde encaminharam ela para X [município onde realiza o tratamento oncológico]. E aí ela foi para casa e depois voltou de novo para cá [...] umas três vezes e com esta agora está inteirando três vezes que ela fica internada com esta mesma dor. [...] Não dava nem uma semana que ela chegava em casa e já voltava para o hospital (P4, irmã).

Fui duas vezes com ele para a UPA [Unidade de Pronto Atendimento]. E a dor voltava, era a conta de passar o efeito do remédio e voltava a dor. Nas outras vezes eu nem levei [para a UPA] porque não estava adiantando. Aí era melhor ficar em casa. [...] Aguardando a consulta (P5, esposa).

Deu uma injeçáo [no Hospital do município de procedência]. Vou falar que na hora melhorou, mas não deu resultado mais para frente. Melhorou na hora. Teve Alta. No outro dia, ela amanheceu com dor de novo. Aí trouxe para cá [Hospital Oncológico] (P13, filho).

Observa-se que os sucessivos retornos à emergência, em curto espaço de tempo para alívio da dor, apontam as fragilidades na assistência ao controle desse sintoma. Assim, estudos devem ser realizados para contemplarem a assistência adequada no controle eficaz da dor.

Segundo revisão bibliográfica abrangente quanto à eficácia das diretrizes da OMS para tratamento da dor no câncer, ressalta que estas auxiliam os médicos no alívio da dor de câncer para a maioria dos pacientes ${ }^{17}$. Apesar de as diretrizes da OMS serem recentes, publicadas em 1986 e reformuladas em 1996, e da simplicidade para serem aplicadas, pode-se indagar se estas sáo usadas adequadamente? Sugere-se que estudos sejam realizados com esse foco.

Dois participantes do sexo masculino, com vínculo familiar de marido, narraram_ser o momento mais difícil do tratamento quando suas esposas tinham dores, sem medicamento em domicílio para aliviar, conforme relato deste participante.

Tem cinco meses que ela está sofrendo de dor. [...] Melhora, quando ela chega em casa dói. [...] É só o efeito do remédio. [...] O mais difícil [do tratamento oncológico] é agora. Dela reclamar as dores (P9, marido). 
O controle efetivo da dor é um dos fenômenos negligenciado na atenção ofertada por parte dos profissionais de saúde. Contudo, o controle da dor em domicílio é um dos pontos essenciais na assistência aos pacientes para promover a dignidade dos sujeitos e o resgate da sua autonomia ${ }^{18}$.

No que tange ao controle eficaz da dor, o relato que segue demonstra o comprometimento do sono e, logo, da qualidade de vida dos atores envolvidos (pacientes e familiares):

E aí ela não dormia e eu também não dormia. Teve um dia que eu não sei se eu dormi ou se eu apaguei... sei que quando eu acordei o sol estava alto. Eu não sei se foi porque eu dormi ou dei uma apagada (P9), marido).

Ao ser perguntado ao participante há quanto tempo ele e a familiar não dormiam em virtude do controle inadequado da dor, este relata:

Uns dois meses. Porque às vezes estava dormindo... acordava no meio da noite, não tinha remédio [para $\mathrm{dor}]$, tinha que esperar ( $\mathrm{P} 9$, marido).

Para o Consenso Brasileiro sobre Manejo da Dor Relacionada ao Câncer, a avaliação da dor deve focar-se na intensidade, na localização, nos fatores que interferem na piora e no alívio do sintoma, nos tratamentos realizados anteriormente e nos tratamentos atuais ${ }^{6}$. Um fator de grande relevância é compreender que a avaliação da dor deve levar em consideração o seu impacto no sono, no desempenho das atividades do cotidiano. Assim, os objetivos do controle da dor focam-se na melhoria da sensação de conforto e no desempenho das atividades do dia a dia.

Apesar do inquestionável ganho para a qualidade de vida do paciente e conforto para os familiares, o sintoma da dor ainda é deixado para um segundo plano, associando-se frequentemente a "um sintoma inerente à história natural da doença”"(137).

Essa associação impacta diretamente na naturalização da dor, como uma consequência inevitável do câncer, sobre a qual não se pode fazer nada, reproduzindo "é assim mesmo, é por causa do câncer avançado", contextualizando o desconhecimento de muitos profissionais de saúde quanto à adequada avaliação da dor e a possibilidades de intervenção. Esse cenário ocasiona aos pacientes e familiares a incansável busca por alívio da dor e do sofrimento, cabendo-lhes somente o lugar "temos que carregar este fardo".

Resta ao paciente suportar a dor e, ao familiar, assistir ao sofrimento intenso ocasionado pela falta da avaliação eficaz e da medicação adequada para controle da dor.
O Brasil precisa avançar em dois pontos: acesso aos medicamentos de dor e capacitação intensiva dos médicos ${ }^{15}$, pois o investimento restrito ao acesso não garante um eficaz controle da dor, uma vez que que o médico necessita ter conhecimento para uma boa avaliação do sintoma e oferecer uma prescrição adequada.

\section{CONCLUSÃO}

A referida pesquisa teve o objetivo de descrever a percepção dos familiares dos pacientes com câncer avançado quanto às dificuldades vivenciadas no acesso aos medicamentos para controle da dor.

Os estudos realizados no Brasil sobre dor no câncer geralmente possuem foco clínico; ou seja, avaliação da dor, uso de medicamentos/opioides. É escassa na literatura a narrativa das pessoas que vivenciam a dor (pacientes/familiares) quanto aos obstáculos no acesso aos medicamentos para analgesia. Desse modo, a pesquisa possui objetivo de evidenciar as limitaçóes no acesso a opioides e demais medicamentos para dor na populaçáo estudada.

Optou-se por realizar a pesquisa com os cuidadores informais e não com os pacientes com câncer avançado, em razão das peculiaridades vivenciadas pelos pacientes no momento do câncer nesse estágio, como dor e demais sintomas frequentes, internaçóes sucessivas, fragilidade emocional. Desse modo, a pesquisa poderia ser um fator estressor para esses indivíduos.

Vale ressaltar que o foco principal da pesquisa foi descrever as dificuldades encontradas no acesso para ao controle da dor, no qual os cuidadores informais poderiam oferecer com propriedade tais informaçóes, tendo em vista serem estes frequentemente os responsáveis por gerenciar e viabilizar tais processos.

Um ponto importante a evidenciar é que, na percepção quanto à dor, a intensidade do sintoma poderia ser diferente do relatado na pesquisa, caso o participante fosse o paciente.

Todos os participantes, familiares dos pacientes com câncer avançado entrevistados narraram a dificuldade no acesso aos opioides e demais medicamentos para a dor. Os obstáculos encontrados foram: dificuldade da dispensação pelo SUS por conta dos trâmites burocráticos; demora em conseguir a liberação do medicamento, sendo o tempo de espera geralmente de três semanas pelo SUS; dificuldade para compra nos municípios de pequeno porte. Todos os participantes relataram comprar os medicamentos para controle da dor, impactando na renda familiar. Outro caminho também relatado para conseguir os medicamentos foi a doação por meio de ONG ou outras instituiçôes de caridade. Os participantes referem que a doação tem sido muito importante para aliviar o impacto 
na renda familiar, possibilitando investir em outras necessidades dos pacientes em tratamento oncológico como alimentação mais saudável.

Os cuidados paliativos, onde se incluí o controle eficaz da dor, são elementos essenciais na cobertura universal de saúde. Assim, torna-se indispensável no Brasil o avanço de uma Política Pública para os pacientes com câncer, na qual seja prioritário o eficaz controle da dor, pautado na facilitação do acesso, mediante dispensação dos opioides e demais medicamentos para analgesia.

Sugere-se que futuros estudos possam utilizarem-se de abordagem qualitativa e quantitativa com populaçóes de características sociodemográficas diferenciadas, associando-as ao acesso a opioides e demais medicamentos para dor, e de técnicas de coletas de dados que selecionem pacientes com diferentes tipos de câncer e estágios da doença, e tempo de uso de medicamentos para dor.

Ressalta-se a relevância, na formação, da capacitação que priorize a abordagem da dor, como um imperativo ético que transversalize a assistência realizada por todos os profissionais de saúde. Somente, desse modo, será possível intervir no "mar de sofrimento", no não abandono, na inequidade, e promover a dignidade humana para os que vivenciam a dor, o sofrimento diante de uma doença que ameaça a continuidade da vida.

\section{CONTRIBUIÇÕES}

Francine Rosa Portela foi responsável pela concepção da pesquisa, trabalho de campo e redação do artigo. Celina Maria Modena orientou a pesquisa e fez a revisão do manuscrito.

\section{DECLARAÇÃO DE CONFLITO DE INTERESSES}

Nada a declarar.

\section{REFERÊNCIAS}

1. Knaul FM, Farmer PE, Krakauer EL, De Lima L, Bhadelia A, Jiang Kwete X, Arreola-Ornelas H, et al. Alleviating the access abyss in palliative care and pain relief - an imperative of universal health coverage: the Lancet Commission report. The Lancet. 12 de outubro de 2017; 391(10128): 1391-1454.

2. Horton R. Offline: A sea of suffering. The Lancet. 2018; 391(10129): 1465.

3. Associação Latinoamericana de Cuidados Paliativos. Indicadores de Cuidado Paliativo ALCP. Houston: IAHPC Press; 2013.

4. World Health Organization. Palliative Care [internet] Genebra: WHO; 2017 [acesso em 2018 apr 17].
Disponível em: http://www.who.int/mediacentre/ factsheets/fs402/en/.

5. Instituto Nacional de Câncer. Cuidados paliativos oncológicos: controle da dor. Rio de Janeiro: INCA; 2001.

6. Wiermann EG, Diz MPE, Caponero R, Lages PSM, Araujo CZS, Castro Bettega RT, et al. Consenso Brasileiro sobre Manejo da Dor Relacionada ao Câncer. RBOC. 2014; 10(38): 132-143.

7. Victor GHGG. Cuidados Paliativos no Mundo. RBC. 2016; 62(3): 267-270.

8. Associação Latinoamericana de Cuidados Paliativos. Atlas de Cuidados Paliativos de Latinoamérica ALCP. Houston: IAHPC Press; 2012.

9. Organisation for the Prevention of Intense Suffering. La morfina como derecho humano. [internet]. Genebra: OPIS; 2018 [acesso em 2018 abr. 19]. Disponível em: http://infocuidadospaliativos.com/wp-content/ uploads/2018/03/la-morfina-como-derecho-humano.pdf.

10. Minayo MCS. Pesquisa Social. Teoria, método criatividade. 18. ed. Petrópolis: Vozes; 2001.

11. Flick U. Introdução à pesquisa qualitativa. 3. ed. Porto Alegre: Artmed; 2009.

12. Boni V, Quaresma SJ. Aprendendo a entrevistar: como fazer entrevistas em Ciências Sociais. Em tese. 2005; 2(1): 68-80.

13. Bardin L. Análise de Conteúdos. São Paulo: Martins Fontes; 1976.

14. Miaskowski C, Zimmer EF, Barrett KM, Dibble SL, Wallhagen M. Differences in patients and family caregivers perceptions of the pain experience influence patient and caregiver outcomes. Pain. 1997;72(12):217-26.

15. Instituto Nacional de Câncer José de Alencar Gomes da Silva. Dor e Burocracia: Assistência: apesar de diretriz da declaração mundial contra o câncer para o controle da dor, acesso de paciente à morfina ainda é difícil. Revista Rede Câncer. 2014; (25):14-17.

16. Economist Intelligence Unit. The 2015 Quality of Death index: ranking palliative care across the word. London: Economisti Intelligence Unit; 2015.

17. Carlson CL. Effectiveness of the World Health Organization cancer pain relief guidelines: an integrative review. J Pain Res. 2016; 9:515-534.

18. Oliveira Júnior AA. Silêncio. Belo Horizonte: O lutador; 2009. 